\title{
Editorial
}

\section{Optical spectroscopic markers of cancer}

\author{
V. Backman ${ }^{\mathrm{a}}$ and H.K. Roy ${ }^{\mathrm{b}}$ \\ ${ }^{a}$ Biomedical Engineering Department, Northwestern University, Evanston, IL, USA \\ ${ }^{\mathrm{b}}$ Department of Internal Medicine, Evanston Northwestern Healthcare, Evanston, IL, USA
}

Welcome to the Special Issue on Optical Spectroscopic Markers of Cancer. Disease markers have classically encompassed proteins, gene expressions, proteomics and histological abnormalities (dysplasia). It is increasing being realized that optical properties of the tissue can provide fundamental insights into tissue properties at structural and molecular levels and, hence, serve as novel markers of disease. Biophotonics is an emerging field that bridges biomedical optics to medicine. A particularly exciting application of biophotonics is on the diagnosis, detection, and screening for cancer. Recently, noninvasive or minimally invasive biophotonics techniques have been developed that sense changes in tissue at molecular, subcellular, microscopic and macroscopic levels. These optical markers are indicative of alterations in tissue morphology, chemical composition as well as functional changes such as changes in microvasculature. In some cases, optical markers have specific histological determinants. In these cases biophotonics techniques may provide a less invasive and fast means of identifying dysplasia (the "optical biopsy"). Other groups have focused on using optical techniques to assess the genetic/epigenetic correlates of field carcinogenesis and thus these spectral markers can be used for riskstratification.

The purpose of this issue is to highlight the spectrum of approaches and the recent advances in the field of optical markers of carcinogenesis. Articles in this issue discuss the following biophotonics techniques: i) light absorption spectroscopy for sensing specific molecules in tissue such as hemoglobin, water and lipids (Kukreti et al.). ii) Elastic light scattering for charaterization of subcellular and tissue morphology (Wax et al., Gong et al. and Turzhitsky et al.). iii) Raman spectroscopy for analysis of molecular composition of tissue (Keller et al.). These papers report the optical markers of neoplastic cells and tissues (Kukreti et al., Wax et al., and Gong et al.) as well as the markers of the field-effect, i.e. alterations in tissue located outside of cancerous sites that are otherwise undetected by conventional histology (Keller et al. and Turzhitsky et al.). Finally, these papers cover a wide range of organ sites including breast, gastrointestinal tract, cervix, skin, and brain. We hope you will find this Special Issue to be a useful introduction that will stimulate further interest in the development of optical markers for cancer screening and diagnosis. 


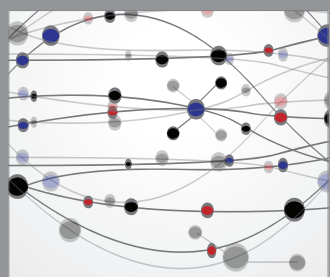

The Scientific World Journal
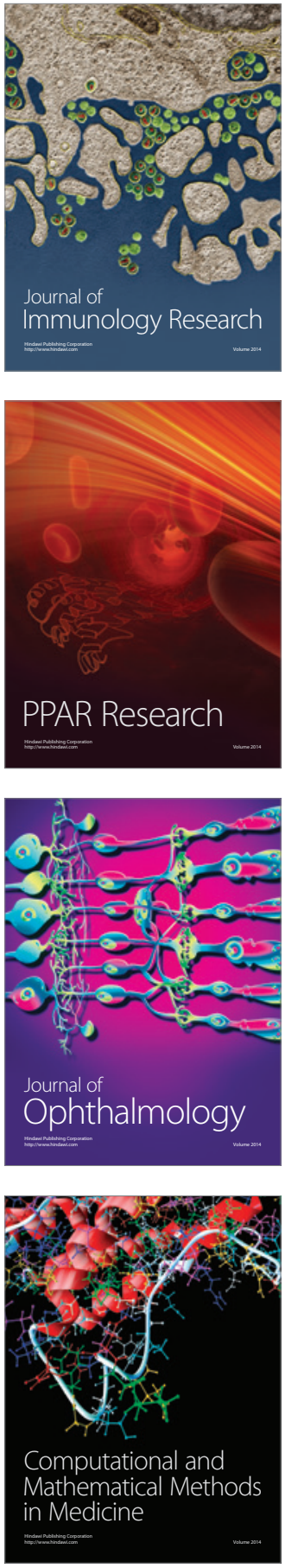

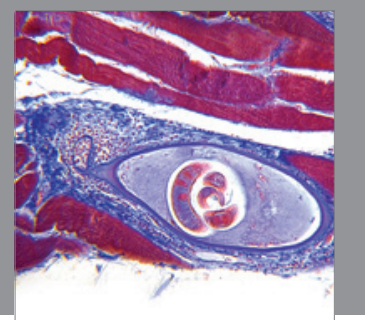

Gastroenterology

Research and Practice
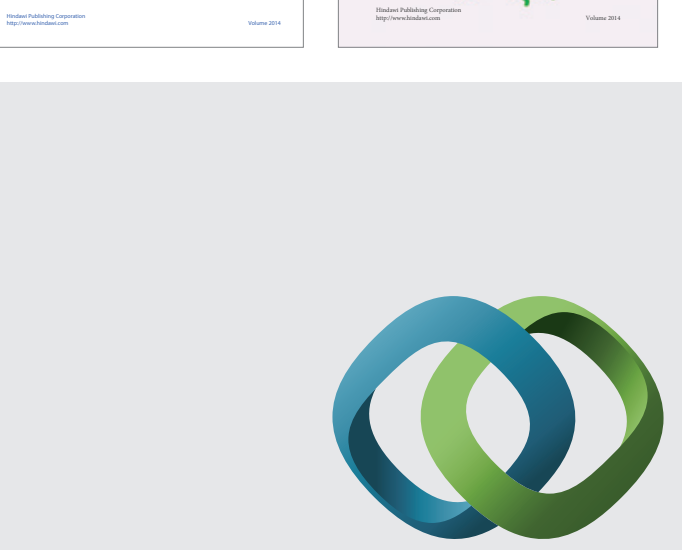

\section{Hindawi}

Submit your manuscripts at

http://www.hindawi.com
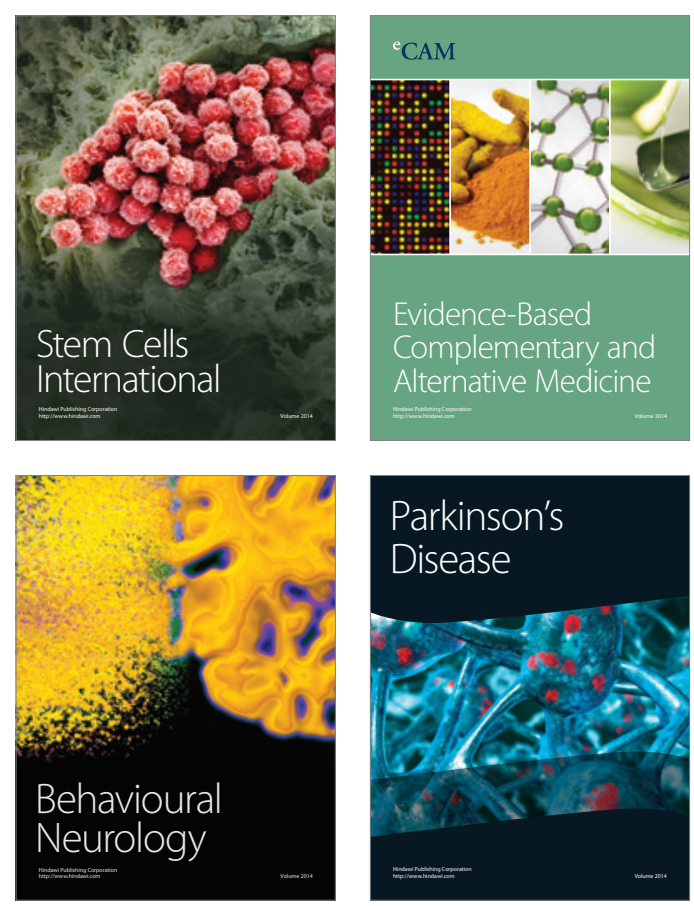

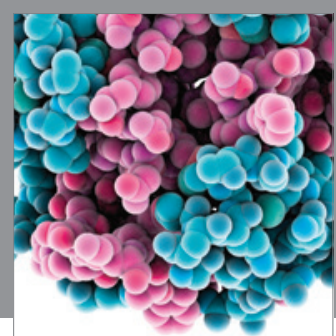

Journal of
Diabetes Research

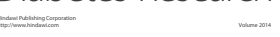

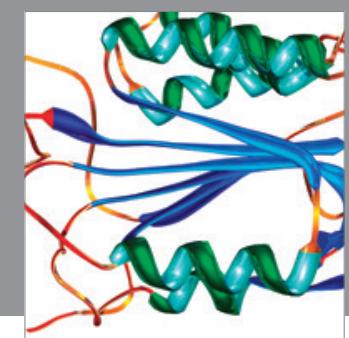

Disease Markers
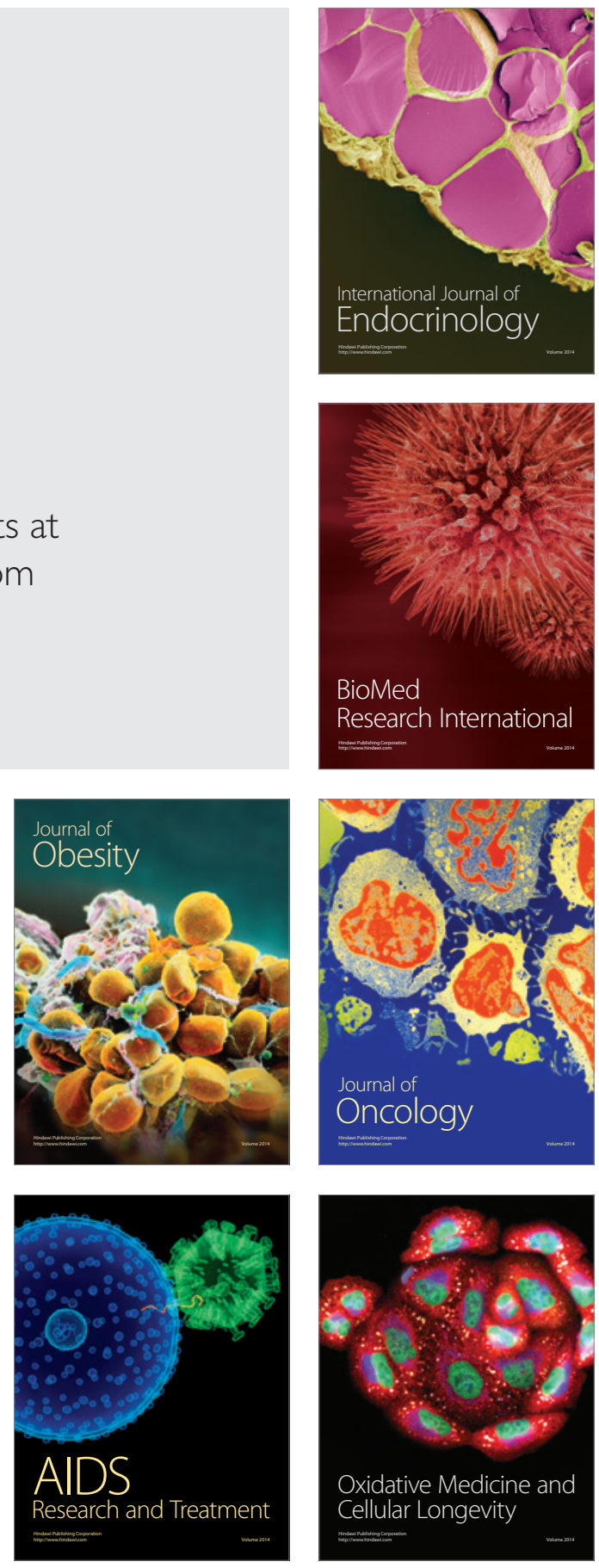